\title{
IR detection of thermal waves - effect of imaging conditions on the background fluctuation limit
}

\author{
by J. Bolte, B.K. Bein, J.Pelzl
}

Institute for Experimental Physics III, AG Solid State Spectroscopy, Ruhr-Universitat Bochum D-44780 Bochum, FR Germany

\begin{abstract}
Starting from the basic principles of photon detection, a general theoretical description is here given for the incoherent background fluctuation limit of thermal wave detection. Different imaging conditions of IR detection of thermal waves and different detectors are considered. The theoretical limits are compared with measurements obtained for a MCT detector. Good agreement between the observed detection limits and the theoretical prediction is obtained.
\end{abstract}

\section{Introduction}

Thermal waves, excited in solids by intensity-modulated heating, can be used to determine thermophysical parameters, e.g. the thermal diffusivity and effusivity. Since the penetration depth of thermal waves decreases with the modulation frequency of heating, depth-selective information about the thermal properties can be obtained by measuring the amplitude and phase as functions of the modulation frequency. In general, thermal wave measurements are nondestructive and in the case of IR detection of the thermal wave response, thermal waves are most appropriate for non-contact remote measurements of thermal properties and thermal depth profiles. The limit of detection of thermal waves for a given measurement setup, is affected by the total noise of the setup within the measured bandwidth. The total noise is mainly caused by the noise produced in the detector itself, the noise of the electronic system following the detector, and the noise of the incident radiation to which the detector responds. The ultimate limits of detection are set by the fluctuations of the incident radiation, which can be identified, when a cooled detector with low internal noise and a low-noise preamplifier are used. There are two types of fluctuations of the incident radiation: fluctuations of the signal radiation and fluctuations of the background radiation. For the detection of thermal waves, usually the fluctuations in background radiation are dominant and the fluctuations in signal radiation are negligible.

\section{Background fluctuations}

The small variations of the detector signal which correspond to the thermal wave response are distinguished from the background radiation level by filtering the detector signal with the help of a lock-in amplifier at the modulation frequency $f$ of the thermal wave. Nevertheless, the detection is affected by incoherent and coherent fluctuations:

- Coherent fluctuations may be due to secondary thermal waves produced in the components of the IR optics (lenses and filters) by the modulated laser beam used for the excitation of the thermal wave or by the thermal wave response itself [1]. Since the thermal wave signal depends on the stationary temperature $T$, coherent noise may also arise from slow changes in the stationary temperature of the sample [2]. 
- Incoherent noise originates from fluctuations of the background radiation incident on the detector. The background radiation can come from the focused sample surface area, from the surroundings of the sample as well as from the components of the IR optics.

The signal-to-noise ratio due to incoherent background and signal radiation for an infrared detector - whether photoconductive or photovoltaic - can be derived fom the basic principles of photon detection $[3,4]$ and is given by

$$
\frac{S}{N}=\frac{\bar{i}_{s}^{2}(v)}{\overline{i(v)_{N}{ }^{2}}}=\frac{\eta(v) P_{s}^{2}(v)}{a B h v\left[P_{s}(v)+P_{b}(v)\right]}
$$

Here $i_{s}(v)$ is the average signal current, $\overline{i(v)_{N}^{2}}$ are the mean square current fluctuations, $P_{s}(v)$ is the power of the incident signal radiation of frequency $v, P_{b}(v)$ is the background radiation and $\eta(v)$ is the quantum efficiency. $B$ is the filter bandwidth and $a$ is a constant, which is 2 in the case of photovoltaic detection and 4 for photoconductive detection [5].

When thermal waves are measured, the power of the incident signal radiation $P_{s}(v)$ is the power related to the thermal wave $\delta T$. It can be described by the derivative with respect to the temperature of that part of the radiation originating from the sample surface of time-averaged sample temperature $T[4,6]$

$$
P_{s}(v)=\frac{\partial P_{s a m}(v)}{\partial T} \delta T
$$

In photothermal measurements, thermal waves usually vary in the range from some milliKelvin up to 1 or $2 \mathrm{~K}$, in comparison to average sample temperatures, respectively background temperatures of 300 to $1000 \mathrm{~K}$. Thus the power related to the thermal wave is small in comparison to the power of the background radiation, and after inserting equation (2) into equation (1), we obtain the expression

$$
\frac{S}{N}=\frac{\eta(v)\left[\partial P_{s a m}(v) / \partial T\right]^{2}}{a B h v P_{b}(v)}(\delta T)^{2}
$$

for the signal-to-noise ratio, which can be resolved for the quantity $\delta T$

$$
\delta T=\frac{\sqrt{a B h v P_{b}(v)}}{\sqrt{\eta(v)}\left[\partial P_{s a m}(v) / \partial T\right]} \sqrt{\frac{S}{N}}
$$

and which can be identified with the minimal detectable thermal wave or noise-equivalent thermal wave

$$
N E \delta T=\delta T_{S / N=1}=\frac{\sqrt{a B h v P_{b}(v)}}{\sqrt{\eta(v)}\left[\partial P_{s a m}(v) / \partial T\right]}
$$

For an exact calculation of the minimal detectable thermal wave amplitude $N E \delta T$, the technical parameters of the detection system have to be described in detail, mainly the imaging conditions, which are essential for an exact description of the collected and focused power incident on the detector. We now consider an IR system according to figure 1 . The thermal radiation of the heated sample surface is focused onto the infrared detector by only one infrared lens. The lens is positioned at half the distance between sample and detector to obtain a $2 \mathrm{f}-$ image, where $f$ is the focusing length of the IR lens. In this case the detected spot area is equal 
to the sensitive area of the detector. $\vartheta_{L}$ is the angle between the surface normal of the detector element and the outer edge of the infrared lens and $\vartheta_{D}$ is half of the detector's field of view. A cut-on filter positioned just in front of the detector blocks the reflected light of the heating laser to avoid coherent noise. For such a focusing system, the background radiation $P_{b}(v)$ incident on the detector is the sum of the following contributions:

- the radiation emitted in the frequency interval $v, v+d v$ by the detected sample surface area of temperature $T$ within the focused solid angle. Here the emissivity of the sample surface is assumed to be $\varepsilon_{s}=1$.

$$
P_{\text {sam }}(v)=2 \pi A_{D} F_{F}(v) F_{L}(v) w(v, T) \mathrm{d} v \int_{0}^{\vartheta_{L}} \cos \vartheta \sin \vartheta \mathrm{d} \vartheta
$$

- the radiation from the surroundings, which are at a stationary temperature $T_{0}$, striking the detector, if the solid angle of the focused object is smaller than the maximum solid angle of the detector given by the field of view, $\vartheta_{L}<\vartheta_{D}$

$$
P_{s u r}(v)=2 \pi A_{D} F_{F}(v) w\left(v, T_{0}\right) \mathrm{d} v \int_{\vartheta_{L}}^{\vartheta_{D}} \cos \vartheta \sin \vartheta \mathrm{d} \vartheta
$$

- the thermal radiation emitted by the IR lens, which has got the temperature $T_{0}$, and the radiation from the surroundings which is reflected by the lens onto the detector

$$
P_{L}(v)=2 \pi A_{D} F_{F}(v)\left[1-F_{L}(v)\right] w\left(v, T_{0}\right) \mathrm{d} v \int_{0}^{\vartheta_{L}} \cos \vartheta \sin \vartheta \mathrm{d} \vartheta
$$

- the radiation emitted by the cut-on filter, which is assumed to be at the temperature $T_{0}$, and the radiation from the surroundings which is reflected by the filter onto the detector

$$
P_{F}(v)=2 \pi A_{D}\left[1-F_{F}(v)\right] w\left(v, T_{0}\right) \mathrm{d} v \int_{0}^{\vartheta_{D}} \cos \vartheta \sin \vartheta \mathrm{d} \vartheta
$$

Here $F_{F}$ is the transmittance of the filter, $F_{L}$ that of the IR-lens and $w(v, T)$ is Planck's blackbody law. We assume that the detector dewar is at a much lower temperature than that of the object field, so that the radiation of the dewar can be neglected. It is also neglected that part of the reflected radiation may arise from the cooled detector dewar. The sum of the background radiations of different origins incident on the detector is then calculated to be

$$
\begin{aligned}
P_{b}(\lambda)=A_{D} \frac{4 \pi h c^{2}}{\lambda^{5}}\left\{\left(F(\lambda) \frac{\mathrm{d} \lambda}{e^{\frac{h c}{\lambda k T}}-1}+[1-F(\lambda)] \frac{\mathrm{d} \lambda}{e^{\frac{h c}{\lambda k T_{o}}}-1}\right) \int_{0}^{\vartheta_{L}} \cos \vartheta \sin \vartheta \mathrm{d} \vartheta\right. \\
\left.+\frac{\mathrm{d} \lambda}{e^{\frac{h c}{\lambda k T_{o}}}-1} \int_{\vartheta_{L}}^{\vartheta_{L}} \cos \vartheta \sin \vartheta \mathrm{d} \vartheta\right\}
\end{aligned}
$$

where now the power is written as a function of wavelength and where $F(\lambda)$ is the transmission of the whole IR optics $\left(F(\lambda)=F_{L}(\lambda) \cdot F_{F}(\lambda)\right)$. Following equation (5) the minimal detectable thermal wave in the case of integral detection over a wavelength interval $\lambda_{1}<\lambda<\lambda_{2}$ is:

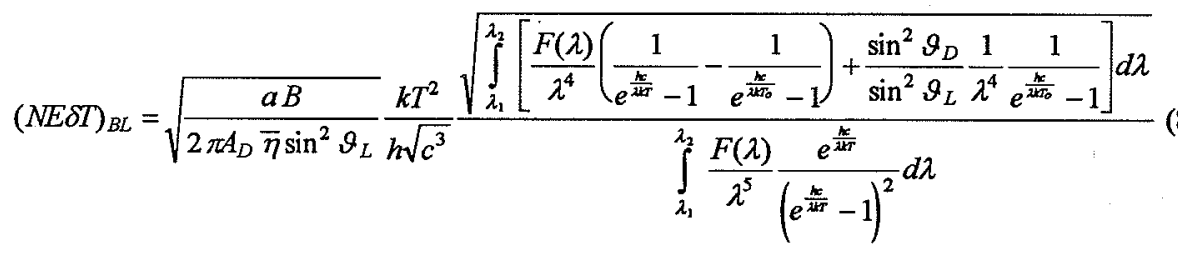


As can be seen from equation (8), the detection limit depends on the average sample temperature $T$ and on the temperature of the surroundings $T_{0}$. Furthermore it depends on technical parameters characterizing the detection system: the detector sensitive area $A_{D}$, the effective quantum efficiency $\bar{\eta}$, the wavelength interval $\lambda_{1}<\lambda<\lambda_{2}$ of the detector, the filter bandwidth $B$ used for electronic filtering of the thermal waves from the radiation background, the transmittance $F(\lambda)$ of the IR optics and the angles $\vartheta_{L}$ and $\vartheta_{D}$ described in figure 1 .

\section{Effects of detector characteristics and imaging conditions}

In figure 2 results of equation (8) are plotted versus the stationary temperature $T$ of the sample for different IR detectors, namely a photoconductive MCT detector (cut-off wavelength $\left.\lambda_{2}=12 \mu \mathrm{m}\right)$, a photovoltaic $\operatorname{lnSb}$ detector $\left(\lambda_{2}=5.4 \mu \mathrm{m}\right)$ and a photovoltaic $\ln A s$ detector $\left(\lambda_{2}=3.3 \mu \mathrm{m}\right)$. For the field of view, the detector area and the quantum efficiency values of $\left(2 \vartheta_{D}\right)=60^{\circ}, A_{D}=2 \mathrm{~mm}^{2}$ and $\eta(\lambda)=1$ have been assumed. The IR optics consist of a silicon filter and $a \mathrm{CaF}_{2}$ lens with a focal length of $\mathrm{f}=100 \mathrm{~mm}$ and a diameter of $d=100 \mathrm{~mm}$ leading to an angle for the focused signal radiation of $\vartheta_{L}=28^{\circ}$. The temperature of the IR optics and of the surroundings are assumed to be $T_{0}=300 \mathrm{~K}$, whereas the detector is cooled. The bandwidth is $B=1 \mathrm{~Hz}$.

It can be seen that the minimal detectable thermal wave decreases with increasing temperature. Below ambient temperature the MCT detector is most suitable for the detection of thermal waves and above ambient temperature the InSb detector is able to detect smaller temperature oscillations than the others. At ambient temperature $(300 \mathrm{~K})$, the MCT detector and the InSb detector both lead to nearly the same noise equivalent thermal wave of $(N E \delta T)_{B L} \approx \mathbf{3 3} \mu K$. This can be explained by the peak wavelength of the signal radiation spectrum of thermal waves according to equation (2). For temperatures below $300 \mathrm{~K}$ the maximum signal radiation is out of the spectral range of the $\operatorname{lnSb}$ and $\ln A s$ detector, whereas at higher sample temperatures the maximum spectral signal radiation is below $6.8 \mu \mathrm{m}$. The MCT detector detects the high background radiation level, whose peak wavelength is in the spectral range from $6 \mu \mathrm{m}$ to $10 \mu \mathrm{m}$ for temperatures between $300 \mathrm{~K}$ and $500 \mathrm{~K}$, whereas the InSb and the InAs detector do not respond to that part of the radiation spectrum. Furthermore it can be seen, that the InAs detector is not suitable for the detection of thermal waves in the temperature range below about $800 \mathrm{~K}$.

In figure 3 , the noise-equivalent thermal wave is plotted versus the average sample temperature for $\mathrm{BaF}_{2}$ and $\mathrm{CaF}_{2}$ lenses. In the case of a MCT detector, it is better to use a $\mathrm{BaF}_{2}$ lens, because its transmittance is better adapted to the spectral range of this detector.

Figure 4 shows the noise equivalent thermal wave versus the field of view $\left(2 \vartheta_{D}\right)$ for a MCT detector at several sample temperatures. The technical parameters are the same as above. It can be seen that it is important to use a detector with a field of view not greater than $\left(2 \vartheta_{L}\right)$, at least for temperatures below about $400 \mathrm{~K}$.

\section{Experimentally observed detection limits}

The minimal detectable temperature oscillation can be determined experimentally by frequency-dependent measurements of thermal waves with a sample, whose thermal and optical properties are well known. Since the measured amplitude contains the noise of the measurement system, a signal to noise ratio $S / N=1$ is reached, if the measured amplitude of the thermal wave is two times the measured noise. This condition leads to the critical frequency of 
the thermal wave for the measured sample. By considering the equation for the temperature oscillation of a semi-infinite homogeneous sample, the minimal detectable temperature oscillation, which is the thermal wave at the critical frequency $f_{(N E \delta r)_{K x}}$ can be estimated by

$$
(N E \delta T)_{E x}=\frac{\eta I_{0}}{2 e \sqrt{2 \pi f_{(N E \delta T)_{E x}}}}
$$

The quantity $e$ in equ. (9) is the effusivity and $\eta$ the absorptivity of the heated sample. $l_{0}$ is the intensity of the heating beam. Figure 5 shows the experimentally observed detection limits for samples of V2A steel and glassy carbon, which are compared with the background fluctuation limit according to equation (8). The minimal thermal wave amplitude resolved with the help of a $\mathrm{HgCdTe}$ detector is about $\delta T \approx 44 \mu \mathrm{K}$ at $300 \mathrm{~K}, \delta T \approx 15 \mu \mathrm{K}$ at $400 \mathrm{~K}$ and $\delta T \approx 9 \mu \mathrm{K}$ at $500 \mathrm{~K}$. Good agreement between the observed detection limits and the theoretical prediction is obtained and one can conclude that the ultimate limit of detection has nearly been reached with the experimental arrangement used here.

\section{Conclusions}

Based on the equation for the noise-equivalent minimal detectable thermal wave, the equipment for IR detection of thermal waves can be adapted to a specific detection task and the focusing conditions can be optimized. Thus the expenses for an IR detection system of thermal waves can be reduced.

Acknowledgements: In part, this work has been supported by the Federal Ministry of Defense

\section{REFERENCES}

[1] MENSING (A:).- IR-Radiometrie thermischer Wellen an plasma-gespritzten NiCoCrAIYBeschichtungen. Diploma thesis, Ruhr-Universität Bochum, 1993. [in German]

[2] KANSTAD (S. O.).- Experimental aspects of photothermal radiometrie. Can. J. Phys. 64: 1155-1164, 1986.

[3] KEYES (R. J.) (Ed.).- Optical and Infrared Detectors, Topics in Applied Physics. Vol.19, Springer-Verlag Berlin Heidelberg New York, 1977.

[4] KINGSTON (R. H.).- Detection of Optical and Infrared Radiation. Springer Ser. in Opt.Sci., Vol.10, 1978, Springer Verlag Berlin Heidelberg NewYork.

[5] KRUSE (P. W.).- Grundlagen der Infrarottechnik. Verlag Berlin Union, 1970. [in German]

[6] GU (J. H.).- Photothermische Radiometrie für Festkörper bei hohen Temperaturen. Ph.D. thesis, Ruhr-Universität Bochum, 1993. [in German]

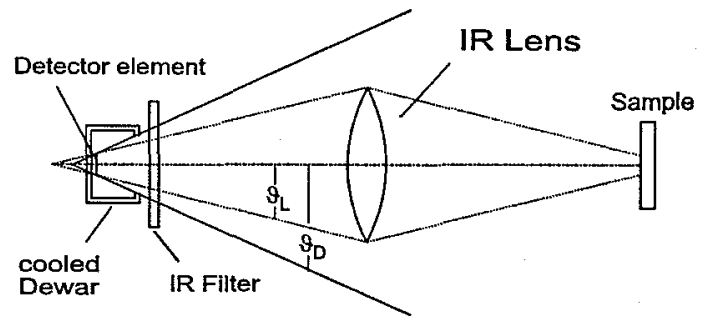

Fig. 1: Imaging conditions 


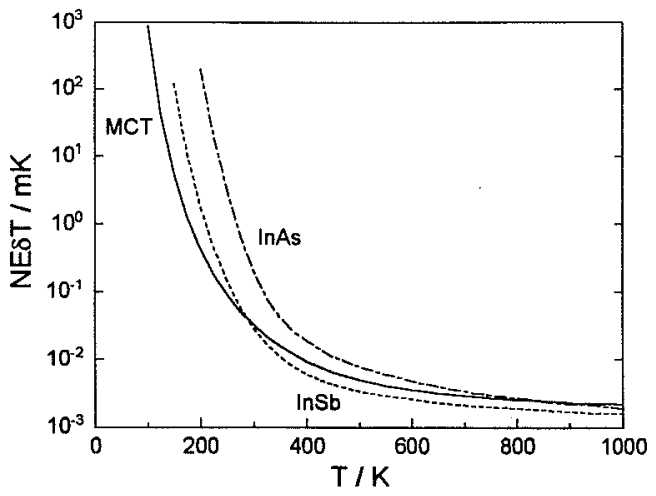

Fig. 2: Noise-equivalent thermal wave for different detectors

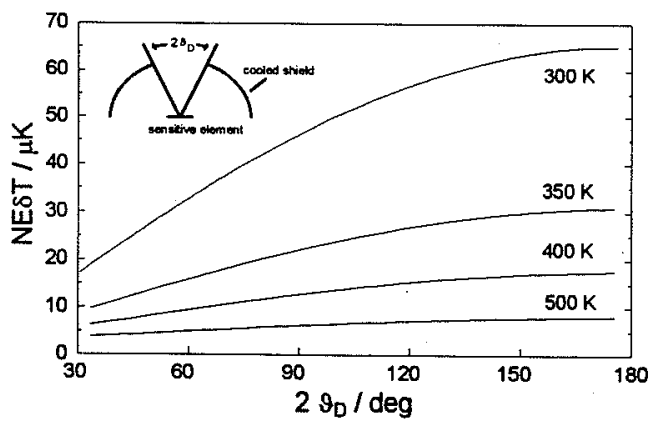

Fig. 3: Noise-equivalent thermal wave for a MCT detector versus the field of view

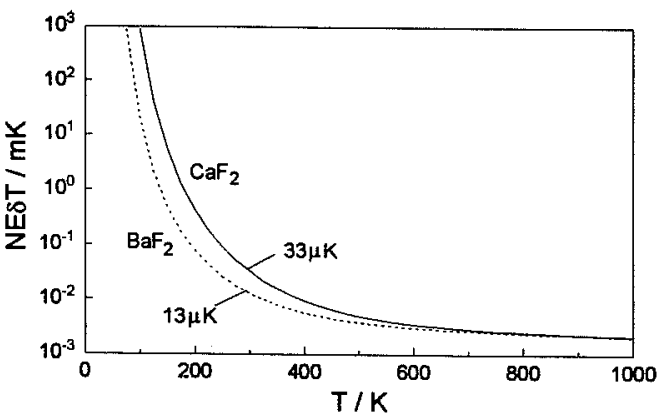

Fig. 4: Noise-equivalent thermal wave for a MCT detector and different IR lenses

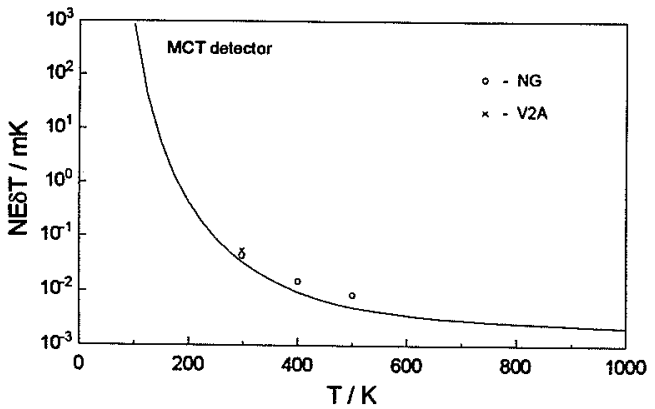

Fig. 5: Noise-equivalent thermal wave for a MCT detector in comparison with some experimentally observed detection limits 